\title{
Budesonide/Formoterol Anti-Inflammatory Reliever and Maintenance or Fluticasone Propionate/Salmeterol Plus as-Needed, Short-Acting $\beta 2$ Agonist: Real-World Effectiveness in patients Without Optimally Controlled asthma (REACT) Study [Corrigendum]
}

Cheng SL, Ho ML, Lai YF, et al. Drug Des Devel Ther. 2020;14:5441-5450.

The authors have advised there is an error in the formatting of the paper title on page 5441, the title "Budesonide/ Formoterol Anti-Inflammatory Reliever and Maintenance or Fluticasone Propionate/Salmeterol Plus As-Needed, Short-Acting $\beta 2$ Agonist: Real-World Effectiveness in pAtients without Optimally Controlled asThma (REACT) Study" should read "Budesonide/Formoterol AntiInflammatory Reliever and Maintenance or Fluticasone Propionate/Salmeterol Plus As-Needed, Short-Acting $\beta 2$ Agonist: Real-world Effectiveness in pAtients without optimally Controlled asThma (REACT) Study".

The authors have also advised the affiliation list on page 5441 is incorrect. The correct author list and affiliation is as follows.

Shih-Lung Cheng, ${ }^{1,2}$ Ming-Lin Ho, ${ }^{3}$ Yun-Fa Lai, ${ }^{4}$ Hao-Chien Wang, ${ }^{5}$ Jeng-Yuan Hsu, ${ }^{6}$ Shih-Feng Liu, ${ }^{7,8}$ Ming-Shyang Huang, ${ }^{9}$ Cheng-Hung Lee, ${ }^{10}$ Ching-Hsiung Lin,,${ }^{11-13}$ LiangWen Hang, ${ }^{14,15}$ Yu-Chih Liu, ${ }^{16}$ Kuang-Yao Yang, ${ }^{17,18, *}$ JiaHorng Wang ${ }^{19, *}$

${ }^{1}$ Department of Internal Medicine, Far Eastern Memorial Hospital, New Taipei City, Taiwan; ${ }^{2}$ Department of Chemical Engineering and Materials Science, Yuan-Ze University, Taoyuan, Taiwan; ${ }^{3}$ Division of Chest Medicine, Kuang Tien General Hospital, Taichung, Taiwan; ${ }^{4}$ Department of Internal Medicine, E-Da Hospital, I-Shou University, Kaohsiung, Taiwan; ${ }^{5}$ Department of Internal Medicine, National Taiwan
University Hospital, Taipei, Taiwan; ${ }^{6}$ Division of Clinical Research, Taichung Veterans General Hospital, Taichung, Taiwan; ${ }^{7}$ Department of Respiratory Therapy, Kaohsiung Chang Gung Memorial Hospital and Chang Gung University College of Medicine, Kaohsiung, Taiwan; ${ }^{8}$ Division of Pulmonary and Critical Care Medicine, Department of Internal Medicine, Kaohsiung Chang Gung Memorial Hospital and Chang Gung University College of Medicine, Kaohsiung, Taiwan; ${ }^{9}$ Department of Internal Medicine, E-DA Cancer Hospital and Kaohsiung Medical University Hospital, Kaohsiung, Taiwan; ${ }^{10}$ Division of Chest Medicine, Department of Internal Medicine, National Cheng Kung University Hospital, College of Medicine, National Cheng Kung University, Tainan, Taiwan; ${ }^{11}$ Division of Chest Medicine, Department of Internal Medicine, Changhua Christian Hospital, Changhua, Taiwan; ${ }^{12}$ Institute of Genomics and Bioinformatics, National Chung Hsing University, Taichung, Taiwan; ${ }^{13}$ Department of Recreation and Holistic Wellness, MingDao University, Changhua, Taiwan; ${ }^{14}$ School of Nursing \& Graduate Institute of Nursing, China Medical University, Taichung, Taiwan; ${ }^{15}$ Sleep Medicine Center, China Medical University Hospital, Taichung, Taiwan; ${ }^{16}$ Department of Internal Medicine, Keelung Chang Gung Memorial Hospital, Keelung, Taiwan; ${ }^{17}$ Department of Chest Medicine, Taipei Veterans General Hospital, Taipei, Taiwan; ${ }^{18}$ Institute of Emergency and Critical Care Medicine, School of Medicine, National Yang-Ming University, Taipei, Taiwan; ${ }^{19}$ Hyperbaric Oxygen Center, Far Eastern Memorial Hospital, New Taipei City, Taiwan

*These authors contributed equally to this work 


\section{Publish your work in this journal}

Drug Design, Development and Therapy is an international, peerreviewed open-access journal that spans the spectrum of drug design and development through to clinical applications. Clinical outcomes, patient safety, and programs for the development and effective, safe, and sustained use of medicines are a feature of the journal, which has also been accepted for indexing on PubMed Central. The manuscript management system is completely online and includes a very quick and fair peer-review system, which is all easy to use. Visit http://www. dovepress.com/testimonials.php to read real quotes from published authors. 